Article

\title{
Aripiprazole Augmentation to Mood Stabilizers for Obsessive-Compulsive Symptoms in Bipolar Disorder
}

\author{
Gabriele Di Salvo ${ }^{1}$, Giuseppe Maina ${ }^{1,2, *}$, Enrico Pessina ${ }^{3}$, Elena Teobaldi ${ }^{1,2}$, Francesca Barbaro ${ }^{3}$, \\ Azzurra Martini ${ }^{3}$, Umberto Albert ${ }^{4}$ and Gianluca Rosso ${ }^{1,2}$ \\ 1 Department of Neurosciences "Rita Levi Montalcini”, University of Turin, 10126 Turin, Italy; \\ gabriele.disalvo@unito.it (G.D.S.); elena.teobaldi@unito.it (E.T.); gianluca.rosso@unito.it (G.R.) \\ 2 Psychiatric Unit, San Luigi Gonzaga University Hospital, 10126 Turin, Italy \\ 3 Mental Health Department of Alba and Bra, 12042 Cuneo, Italy; enricopessina@hotmail.com (E.P.); \\ barbaro.fra@gmail.com (F.B.); azzurramartini@libero.it (A.M.) \\ 4 Psychiatric Section, Department of Medicine, Surgery and Health Sciences, University of Trieste, \\ 34149 Trieste, Italy; ualber@@units.it \\ * Correspondence: giuseppe.maina@unito.it; Tel.: +39-011-902-6504; Fax: +39-19026595
}

Citation: Di Salvo, G.; Maina, G.; Pessina, E.; Teobaldi, E.; Barbaro, F.; Martini, A.; Albert, U.; Rosso, G. Aripiprazole Augmentation to Mood Stabilizers for Obsessive-Compulsive Symptoms in Bipolar Disorder. Medicina 2021, 57, 9. https:// dx.doi.org/10.3390/medicina 57010009

Received: 3 December 2020

Accepted: 22 December 2020

Published: 24 December 2020

Publisher's Note: MDPI stays neutral with regard to jurisdictional claims in published maps and institutional affiliations.

Copyright: (c) 2020 by the authors. Licensee MDPI, Basel, Switzerland. This article is an open access article distributed under the terms and conditions of the Creative Commons Attribution (CC BY) license (https:// creativecommons.org/licenses/by/ $4.0 /)$.

\begin{abstract}
Background and Objectives: Aripiprazole is a first-line agent in the treatment of bipolar disorder (BD) and available data demonstrates its efficacy on clinical symptoms in serotonin reuptake inhibitors-resistant obsessive-compulsive disorder (OCD) patients. Therefore, aripiprazole augmentation to mood stabilizers could represent a promising treatment in BD patients with comorbid OCD. The study examined the efficacy and safety of aripiprazole added to lithium or valproate for the treatment of obsessive-compulsive (OC) symptoms in euthymic BD patients with comorbid OCD. Materials and Methods: This is a 12-week prospective observational study. The efficacy of aripiprazole on OC symptoms was assessed through the mean change of Yale-Brown Obsessive-Compulsive (YBOCS) total score. Tolerability was assessed with the Utvalg for Kliniske Undersogelser (UKU) side effect scale and by reporting adverse events. Results: A total of 70 patients were included in the analyses. The withdrawal rate was $21.4 \%$, mainly due to adverse events. Mean \pm SD final aripiprazole dose was $15.2 \pm 5.3$ in the completer sample $(N=55)$. The Y-BOCS mean score decreased from $24.0 \pm 4.1$ at baseline to $17.1 \pm 4.3$ at 12 weeks. Treatment response rate (Y-BOCS reduction $\geq 35 \%$ ) was $41.8 \%$, while partial response rate (Y-BOCS reduction greater than $25 \%$ but less than $35 \%$ from baseline) accounted for the other $18.2 \%$ of patients. Overall, $91.4 \%$ of completers had at least 1 adverse effect (tremor, tension/inner unrest, reduced duration of sleep, akathisia). No significant differences emerged comparing aripiprazole efficacy and tolerability between patients treated with lithium or valproate. Conclusion: Our findings show that aripiprazole addition to lithium or valproate can reduce OC symptoms in real-world BD euthymic patients.
\end{abstract}

Keywords: aripiprazole; bipolar disorder; OCD; YBOCS; efficacy; adverse events

\section{Introduction}

Obsessive-compulsive disorder (OCD) is one of the most common psychiatric comorbidities in bipolar disorder (BD). In population-based studies, the prevalence of OCD in BD patients was 11-21\% [1-3]. OCD comorbidity impacts the clinical presentation and course of $\mathrm{BD}$, as it is associated with earlier age at onset, increased prevalence of suicide attempts, psychotic symptoms, rapid cycling, alcohol dependence, and greater functional disability [4-9]. Moreover, BD with co-occurring OCD has been found to be associated with polypharmacy and poor treatment response [10].

The treatment of BD-OCD patients is particularly challenging: the serotonin reuptake inhibitors (SRIs), which are the first-line treatment for OCD, can induce switches, mixed states, or cycle acceleration in BD, especially if administered at high doses and maintained for a long time [11,12]. Available data on the treatment of patients with BD and co-occurring 
OCD and anxiety disorders show more evidence of benefit with using BD treatments primarily, thus giving hierarchical priority to $\mathrm{BD}[10,13]$.

Aripiprazole is a second-generation antipsychotic employed in the treatment of BD: several controlled trials showed its efficacy in all illness phases [14] and its use is recommended by the recent guidelines for the management of patients with BD [15,16]. Moreover, aripiprazole has been shown to be more effective than placebo on clinical symptoms and cognitive functioning in OCD patients who had not responded or not fully responded to SRI treatment alone [17-20]. Therefore, aripiprazole augmentation to mood stabilizers could represent a promising treatment in $\mathrm{BD}$ patients with comorbid $\mathrm{OCD}$, but so far, few data are available. Indeed, only one clinical trial was conducted to study the effects of aripiprazole as an adjuvant treatment for obsessive-compulsive (OC) symptoms in the manic phase of $\mathrm{BD}$, showing good efficacy of aripiprazole in the treatment of OC symptoms [21]. To our knowledge, there are no studies that investigated the efficacy on OC symptoms of aripiprazole augmentation to mood stabilizers in euthymic BD-OCD patients.

The present study aims to assess efficacy on OC symptoms and tolerability of the addition of aripiprazole to a mood stabilizer, either lithium or valproate, in patients with remitted $\mathrm{BD}$ and comorbid OCD.

\section{Materials and Methods}

\subsection{Study Design}

The study had a prospective observational design and involved a series of euthymic $\mathrm{BD}$ patients with comorbid $\mathrm{OCD}$, treated with lithium or valproate monotherapy, and prescribed aripiprazole for OC symptoms.

Aripiprazole starting dose and dosage changes during the follow-up period were established according to clinical judgment. The mood stabilizer blood levels were monitored monthly and, if necessary, the dosage was adjusted to comply with the blood therapeutic range (valproate: $50-100 \mathrm{mcg} / \mathrm{L}$; lithium: $0.5-0.8 \mathrm{Mmol} / \mathrm{L}$ ). Each patient was followed-up for 3 months.

Since the observational design of the study, no attempt was made to influence decisions regarding the study treatments and patients received care as usual.

All participants gave written consent before starting treatments. The research was conducted in accordance with the Declaration of Helsinki in its most recent version (64th WMA General Assembly, Fortaleza, Brazil, October 2013). The study was reviewed and approved by the local Ethics Committee (approval code: 7119/Tit:02/Cat:06; approval date: 18 April 2018).

\subsection{Sample}

Study participants were either referred by general practitioners or psychiatrists, or selfreferred to the Mental Health Department of Alba and Bra (Cuneo, Italy) from May 2018 to February 2020.

Patients fulfilling the following criteria were included: (a) aged 18 to 70 years; (b) Diagnostic and Statistical Manual of Mental Disorders Fifth Edition (DSM-5) diagnosis of BD type I and II (American Psychiatric Association, 2013); (c) no acute mood episodes in the last 3 months; (d) no current active suicidal ideation or recent (within 6 months) suicide attempts; (e) current lithium or valproate monotherapy in therapeutic range; (f) DSM-5 diagnosis of comorbid OCD, with a Yale-Brown Obsessive-Compulsive (Y-BOCS) Scale total score of 16 or higher; (g) consent to participate in the study.

Exclusion criteria were: (a) a present or previous diagnosis of schizophrenia or other psychotic disorders; (b) concomitant severe, unstable, active neurological or physical diseases; (c) current substance use disorder, except for nicotine; (d) pregnancy or breastfeeding; (e) other pharmacological treatments than lithium or valproate monotherapy; (f) history of nonresponse or intolerance to aripiprazole. 


\subsection{Assessment and Procedures}

General socio-demographic and clinical information were collected for each subject by looking at medical reports and by directly interviewing the patients. Patients underwent examinations (control visits) during the follow-up period ( 3 months) according to clinical practice.

OC symptoms were assessed by means of the Y-BOCS scale at baseline and the end of the follow-up period. All psychiatric diagnoses and clinical assessments were made by certified psychiatrists with at least five years of experience The likelihood of aripiprazole to improve OC symptoms was assessed evaluating the mean change in YBOCS total score from baseline to endpoint. Moreover, qualitative analyses were performed, calculating rates of responders (Y-BOCS reduction of at least 35\% from baseline) and partial responders (Y-BOCS reduction greater than $25 \%$ but less than $35 \%$ from baseline), according to stages of response recently proposed by Mataix-Cols and colleagues [22].

\subsection{Statistical Analysis}

Subjects' characteristics were summarized as means and SD for continuous variables and as frequencies and percentages for categorical variables.

Given a significance level of 0.05 , the post-hoc power analysis on the sample size of 70 subjects showed a statistical power $>90 \%$ related to Y-BOCS mean scores.

The normality of data distribution was evaluated using the Kolmogorov-Smirnov test. Since the distribution of Y-BOCS total scores at baseline was not normal (KS: 0.133; $p:$ 0.004), the non-parametric Wilcoxon test was used to assess the changes in Y-BOCS total score from baseline to endpoint. For missing values, a "Last Observation Carried Forward" (LOCF) approach was applied.

Further, aripiprazole efficacy and tolerability were compared between two subgroups (patients taking lithium and patients taking valproate), by way of chi-square $(\chi 2)$ for categorical variables (response rates, percentage of adverse events) and Kruskal-Wallis non-parametric test for continuous variables (mean Y-BOCS scores).

The results from every statistical comparison of the treatment groups were presented as 2 -sides $p$ values rounded to 3 decimal places. The criterion for statistical significance in all comparisons was a $p$-value $<0.05$. All statistical analyses were performed by SPSS software version 26.0.

\section{Results}

Seventy patients fulfilled the study criteria and were enrolled. Thirty-three were males $(47.1 \%)$ and the mean age of the sample was $42.6 \pm 13.6$. There was a slight prevalence of patients with BD type II ( $n=37,52.9 \%)$. Forty-five patients $(64.3 \%)$ were treated with lithium and $25(35.7 \%)$ with valproate. All baseline demographic and clinical characteristics of the sample are shown in Table 1.

Fifty-five patients completed the 12-week follow-up period. The dropout rate was $21.4 \%(n=15)$. Reasons for dropout were consent withdrawal $(n=1)$ and the occurrence of adverse events (AEs) $(N=14)$ : AEs that led to dropout are resumed in Table 2. All dropouts were within week 6 .

Patients who completed the 12-weeks observation period showed an improvement in OC symptoms: Y-BOCS mean score significantly decreased at week $12(17.1 \pm 4.3)$ compared to baseline $(24.0 \pm 4.1)$ (Wilcoxon test: $\mathrm{Z}:-6.390, p<0.001)$ (Figure 1$)$.

Likewise, LOCF analysis showed a significant reduction of the Y-BOCS scores from baseline $(25.3 \pm 4.4)$ to the endpoint $(18.0 \pm 4.8)$ (Wilcoxon test: $\mathrm{Z}:-6.485, p<0.001)$. The qualitative analysis highlighted treatment response (Y-BOCS reduction $\geq 35 \%$ from baseline) in 23 patients (41.8\%) and partial response (Y-BOCS reduction greater than $25 \%$ but less than $35 \%$ from baseline) in 10 patients (18.2\%).

During the follow-up period, no mood recurrence was observed in the sample.

AEs experienced by subjects who completed the observation period are shown in Figure 2. 
Table 1. Baseline demographic and clinical characteristics.

\begin{tabular}{cc}
\hline Parameters & $N=70$ \\
\hline Age, years (mean \pm SD) & $42.6 \pm 13.6$ \\
\hline Sex, $n(\%)$ & $33(47.1)$ \\
Male & $37(52.9)$ \\
Female & \\
Marital status, $n(\%)$ : & $33(47.1)$ \\
Never married & $25(35.8)$ \\
Married & $11(15.7)$ \\
Divorced & $1(1.4)$ \\
Widowed & $11.8 \pm 3.5$ \\
\hline Cducational level, years (mean \pm SD) & \\
Currently working, $n(\%)$ & $33(47.1)$ \\
No & $37(52.9)$ \\
\hline Age at onset of bipolar disorder, years (mean \pm SD) & $23.8 \pm 6.3$ \\
\hline Bipolar disorder subtype, $n(\%)$ & $33(47.1)$ \\
I & $37(52.9)$ \\
\hline II & $19.1 \pm 11.8$ \\
\hline Vime from last mood episode, months (mean \pm SD) & $21.7 \pm 13.4$ \\
\hline Mood stabilizer, $n(\%)$ & $45(64.3)$ \\
\hline Duration of illness, years (mean \pm SD) & $25(35.7)$ \\
\hline
\end{tabular}

Table 2. Adverse events leading to drop-out.

\begin{tabular}{cc}
\hline Patients & Reason for Drop-Out \\
\hline P1 & Hyperkinesia logic; Nausea/vomiting \\
P2 & Akathisia; Reduced duration of sleep; Tremor \\
P3 & Tension/Inner unrest; Akathisia; Reduced duration of sleep; Tremor \\
P4 & Tension/Inner unrest; Akathisia; Nausea/Vomiting; Orthostatic dizziness \\
P5 & Tension/Inner unrest; Nausea/Vomiting \\
P6 & Tension/Inner unrest; Reduced duration of sleep \\
P7 & Tension/Inner unrest; Akathisia; Hyperkinesia logic; Concentration difficulties; \\
P8 & Failing memory \\
P9 & Tension/Inner unrest; Reduced duration of sleep \\
P10 & Tension/inner unrest; Akathisia; Reduced duration of sleep; Tremor; \\
P11 & Concentration difficulties \\
P12 & Tremor; Rigidity \\
P13 & Tension/Inner unrest; Hyperkinesia logic \\
P15 & Akathisia \\
\hline Mean \pm SD final aripiprazole dose was 15.2 \pm 5.3 in the completer sample (N = 55).
\end{tabular}

Overall, $90.1 \%$ of completers (50/55) had at least $1 \mathrm{AE}$; the most common side effects reported in the total sample were tremor $(28.6 \%)$, tension/inner unrest $(27.1 \%)$, reduced duration of sleep $(14.3 \%)$, and akathisia $(11.4 \%)$. 


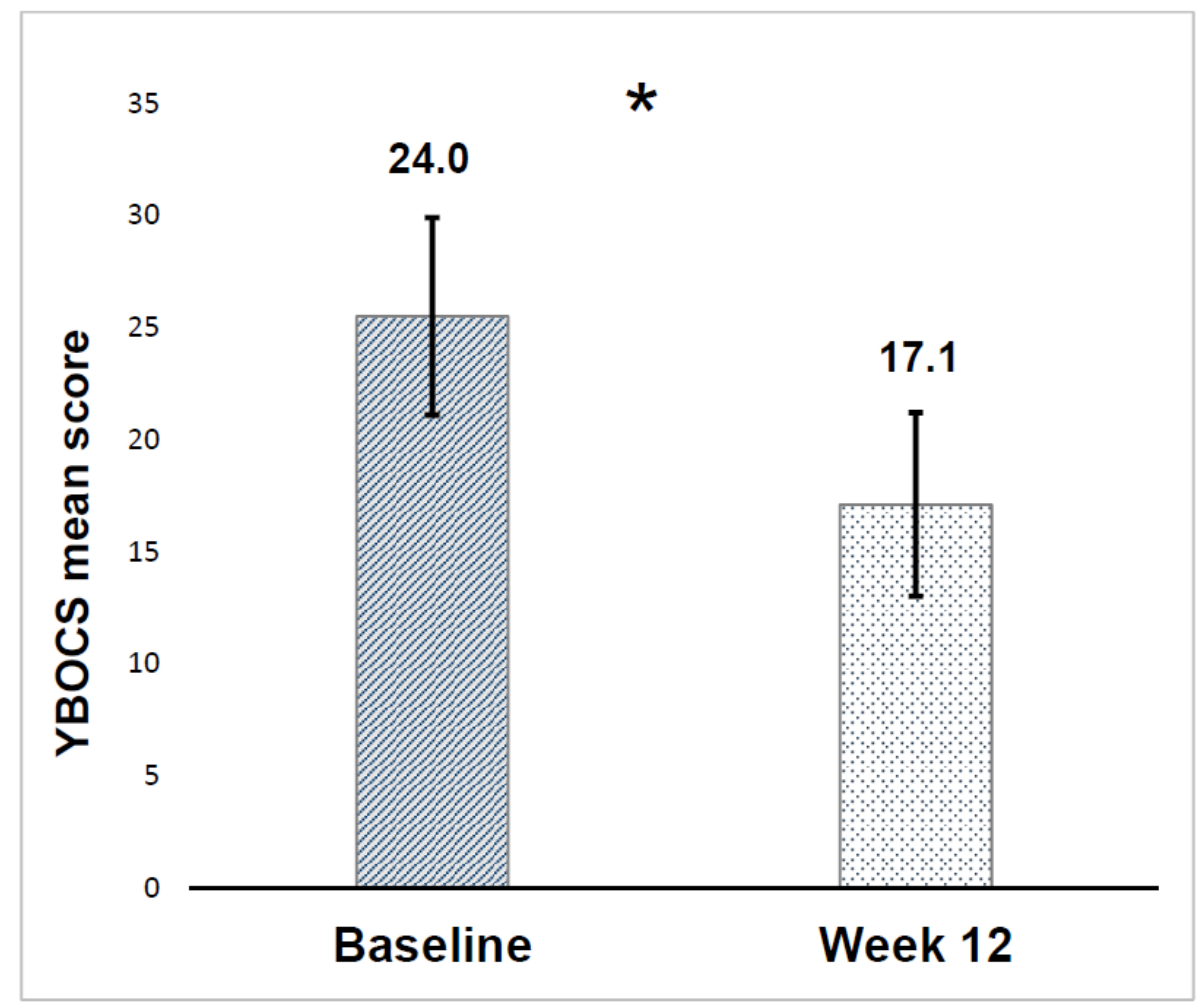

Figure 1. Yale-Brown Obsessive-Compulsive (YBOCS) score mean reduction. ${ }^{*}: p<0.001$.

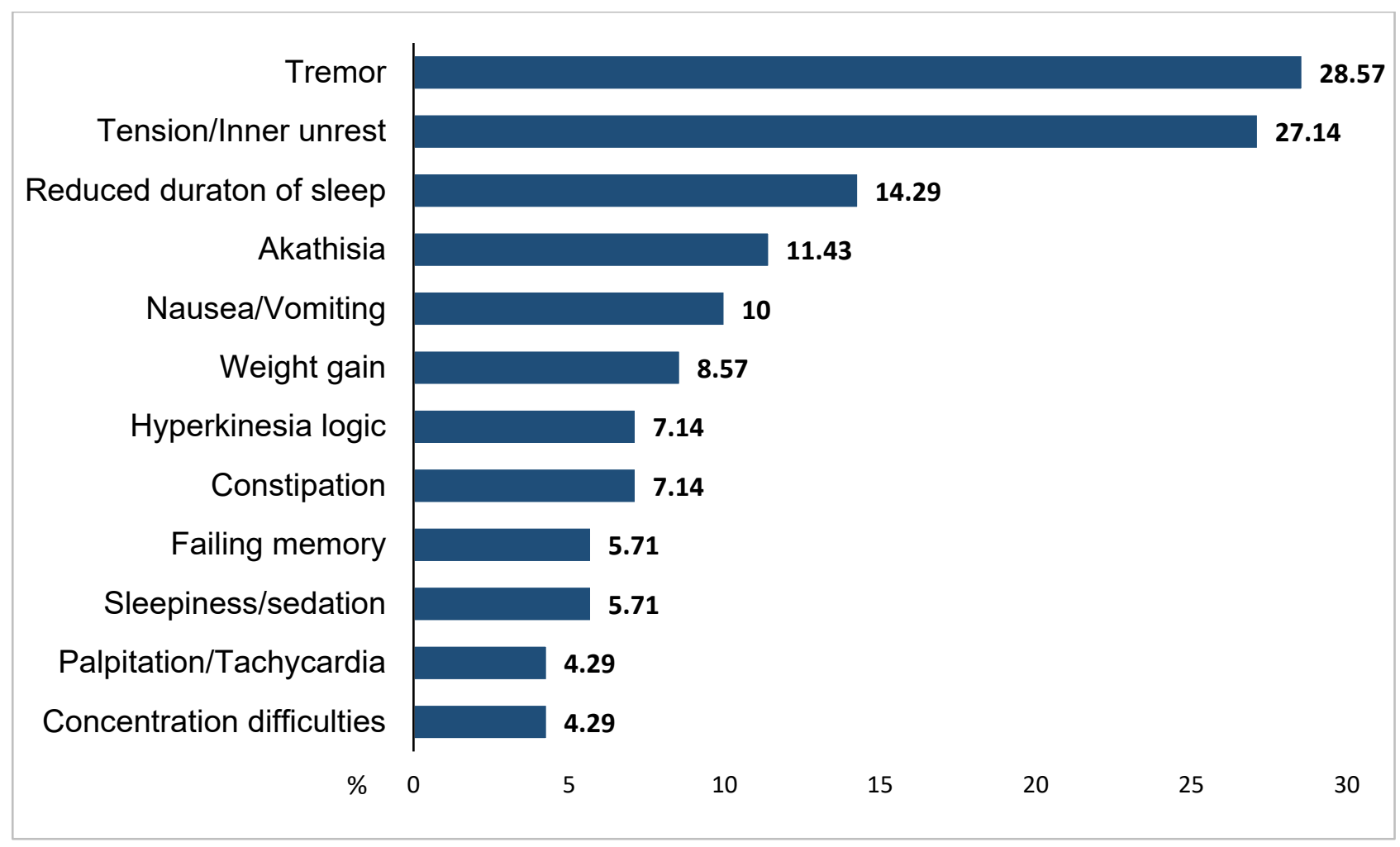

Figure 2. Most common adverse events in completers $(N=55)$. Adverse events $<4 \%$ : asthenia/lassitude/increased fatigability, orthostatic dizziness, weight loss, rigidity, orgastic dysfunction, micturition disturbances, erectile dysfunction, ejaculatory dysfunction, paraesthesias, increased tendency to sweating, increased dream activity, diarrhea, increased sexual desire, polyuria/polydipsia. 
The total sample was further analyzed according to whether patients were treated with lithium $(N=36)$ or valproate $(N=19)$. Analyzing completers, lithium and valproate subgroups did not significantly differ in Y-BOCS scores at baseline (23.9 \pm 4.2 vs. $24.3 \pm 3.9$; $p$ : 0.804$)$ and week $12(17.1 \pm 4.8$ vs. $17.0 \pm 3.5 ; p$ : 0.986). Further, a significant decrease from baseline to week 12 was observed both in the lithium subgroup $(\mathrm{Z}:-5.166, p<0.001)$ and in valproate subgroup (Z: $-3.793, p<0.001)$. Concerning the qualitative analysis, we did not find significant differences between the 2 subgroups, neither examining response rates (lithium subgroup: 41.7\%; valproate subgroup: 42.1\%; p: 0.975), nor considering partial response rates (lithium subgroup: $16.7 \%$; valproate subgroup: $21.1 \%$; $p$ : 0.134 ). Results did not change after LOCF analysis. Lastly, with regards to tolerability, patients with at least $1 \mathrm{AE}$ were $40(89.0 \%)$ in the lithium subgroup and $24(96.0 \%)$ in the valproate subgroup ( $p$ : 0.309).

\section{Discussion}

To our knowledge, this is the first study focusing on the efficacy and tolerability of aripiprazole augmentation to mood stabilizers for the treatment of OC symptoms in euthymic BD patients.

In line with a hierarchical model explaining comorbid psychiatric disorders in BD patients as epiphenomena of the main disease and not as distinct pathologies, we hypothesized that OC symptoms would respond to the addition of a second mood stabilizer. This approach should protect BD patients from the risk of switching into manic or mixed states or of cycle acceleration related to the use of serotonergic agents. Among mood stabilizers, we chose aripiprazole because it's a first-line agent in the treatment of BD and available data demonstrates its efficacy on clinical symptoms in SRI-resistant OCD patients. Aripiprazole dosage during the follow-up period was established and modified according to clinical judgment. Mean final aripiprazole dosage (15.2 mg/day) was in line with doses used both in studies that demonstrated the efficacy of aripiprazole addition in resistant OCD [17-20] and in the only study investigating the efficacy of aripiprazole augmentation in BD-OCD patients [21].

Our findings show that aripiprazole addition to lithium or valproate can reduce OC symptoms in real-world BD euthymic patients. Indeed, in our sample at the end of the 12-week observation period, $41.8 \%$ of subjects met the criteria for treatment response of OC symptoms and $18.2 \%$ showed a partial response, with no difference between patients in lithium or valproate monotherapy. The only study that investigated the efficacy of aripiprazole as an adjuvant treatment for OC symptoms in patients with BD was performed in the manic phase and showed a $>34 \%$ decrease in mean YBOCS score in more than $90 \%$ of subjects treated with aripiprazole $(n=21)$ [21]. However, response rates found in the context of manic or euthymic phases of BD are difficult to compare. Indeed, during an acute manic episode there is a close relationship between OC symptoms and mood elevation; anti-manic agents, including aripiprazole, might be more effective in the treatment of OC symptoms in manic patients rather than in euthymic subjects.

Concerning tolerability, we found high rates of AEs, both in included patients (91.4\%) and in completers $(90.1 \%)$, especially tremor, tension, reduced duration of sleep and akathisia, in line with those most frequently encountered during trials with aripiprazole in patients with BD [23]. Although no severe AEs emerged during the study, one in five patients discontinued the treatment because of the occurrence of side effects. The side effects found in our study are likely to be attributable to the addition of aripiprazole: all adverse events emerged after aripiprazole initiation and there were no differences in tolerability between patients receiving lithium or valproate. However, the impact of drug-drug interactions cannot be excluded. In a multicenter, double-blind, randomized study that evaluated the adjunction of aripiprazole to lithium or valproate as maintenance therapy in BD, the percentage of patients experiencing any AE was lower (75.2\%) [24]. The higher prevalence of AEs found in our real-world patient sample could mainly be 
explained by the fact that strict inclusion and exclusion criteria for randomized controlled trials may exclude the majority of patients encountered in clinical practice [25].

The main limitation of this study is represented by the prospective observational design: our findings require confirmation in double-blind trials. Furthermore, limitations include the relatively small sample size and the absence of a control group. Moreover, we could not run regression models to adjust for potential confounding variables (e.g., baseline YBOSC scores), due to the type of analysis performed and the lack of a control group. Finally, the relatively short length of the observation period (12 weeks) does not allow us to discuss the long-term benefits and risks of this approach; therefore, we aim to continue the follow-up of this cohort for a longer period of time. On the other hand, a point of strength should be taken into account: subjects enrolled for this study were representative of "real-world" patients with BD and comorbid OCD.

\section{Conclusions}

Despite all the limitations, our study is the first to show that a second mood stabilizer, aripiprazole, added to lithium or valproate is effective in reducing OC symptoms in euthymic patients with $\mathrm{BD}$ and co-occurring $\mathrm{OCD}$. Given the high prevalence of $\mathrm{BD}-\mathrm{OCD}$ comorbidity and the negative impact of OCD on clinical course, quality of life, and treatment response of bipolar patients, studies aimed at investigating treatment strategies in this subgroup of difficult-to-treat patients are highly warranted.

Author Contributions: Conceptualization and methodology, G.R., G.M., and U.A.; software, validation, and formal Analysis, G.R., G.D.S., E.P., and E.T.; investigation, resources, and data curation, E.P., F.B., and A.M.; writing — original draft preparation, G.D.S.; writing—review and editing, G.D.S., G.M., G.R., and U.A.; visualization and supervision, all authors. All authors have read and agreed to the published version of the manuscript.

Funding: This research received no external funding.

Institutional Review Board Statement: All participants gave written consent before starting treatments. The research was conducted in accordance with the Declaration of Helsinki in its most recent version (64th WMA General Assembly, Fortaleza, Brazil, October 2013). The study was re-viewed and approved by the AOU San Luigi Gonzaga University Hospital Ethics Committee (approval code: 7119/Tit:02/Cat:06; approval date: (18 April 2018).

Informed Consent Statement: Informed consent was obtained from all subjects involved in the study.

Data Availability Statement: The data that support the findings of this study are not openly available, due to confidentiality of data collected from hospital clinical charts.

Acknowledgments: This research did not receive any specific support from funding agencies in the public, commercial, or not-for-profit sectors.

Conflicts of Interest: Gabriele Di Salvo has been a speaker for Lundbeck and Angelini. Giuseppe Maina is/has been a consultant and/or a speaker and/or has received research grants from Angelini, Boheringer Ingelheim, FB- Health, Janssen, Lundbeck, Otsuka, Innova Pharma. Umberto Albert is/has been a speaker and/or consultant and has received research grants from Angelini, Innova Pharma, Neuraxpharm, Janssen, Lundbeck. Gianluca Rosso is/has been a speaker and/or consultant from Angelini, Janssen, Lundbeck, Otsuka. All other authors declare that they have no conflicts of interest.

\section{References}

1. Chen, Y.W.; Dilsaver, S.C. Comorbidity for obsessive-compulsive disorder in bipolar and unipolar disorders. Psychiatry Res. 1995, 59, 57-64. [CrossRef]

2. Merikangas, K.R.; Akiskal, H.S.; Angst, J.; Greenberg, P.E.; Hirschfeld, R.M.; Petukhova, M.; Kessler, R.C. Lifetime and 12-month prevalence of bipolar spectrum disorder in the National Comorbidity Survey replication. Arch. Gen. Psychiatry 2007, 64, 543-552. [CrossRef] [PubMed] 
3. Faravelli, C.; Abrardi, L.; Bartolozzi, D.; Cecchi, C.; Cosci, F.; D’Adamo, D.; Lo Iacono, B.; Ravaldi, C.; Scarpato, M.A.; Truglia, E.; et al. The sesto fiorentino study: Background, methods and preliminary results. Lifetime prevalence of psychiatric disorders in an Italian community sample using clinical interviewers. Psychother. Psychosom. 2004, 73, 216-225. [CrossRef] [PubMed]

4. Altindag, A.; Yanik, M.; Nebioglu, M. The comorbidity of anxiety disorders in bipolar I patients: Prevalence and clinical correlates. Isr. J. Psychiatry Relat. Sci. 2006, 43, 10-15. [PubMed]

5. Jeon, S.; Baek, J.H.; Yang, S.Y.; Choi, Y.; Ahn, S.W.; Ha, K.; Hong, K.S. Exploration of comorbid obsessive-compulsive disorder in patients with bipolar disorder: The clinic-based prevalence rate, symptoms nature and clinical correlates. J. Affect. Disord. 2018, 225, 227-233. [CrossRef]

6. Magalhães, P.V.; Kapczinski, N.S.; Kapczinski, F. Correlates and impact of obsessive compulsive comorbidity in bipolar disorder. Compr. Psychiatry 2009, 51, 353-356. [CrossRef]

7. Shashidhara, M.; Sushma, B.R.; Viswanath, B.; Math, S.B.; Reddy, Y.C. Comorbid obsessive compulsive disorder in patients with bipolar-I disorder. J. Affect. Disord. 2015, 174, 367-371. [CrossRef]

8. Zutshi, A.; Reddy, Y.C.; Thennarasu, K.; Chandrashekhar, C.R. Comorbidity of anxiety disorders in patients with remitted bipolar disorder. Eur. Arch. Psychiatry Clin. Neurosci. 2006, 256, 428-436. [CrossRef]

9. Di Salvo, G.; Pessina, E.; Aragno, E.; Martini, A.; Albert, U.; Maina, G.; Rosso, G. Impact of comorbid obsessive-compulsive disorder on suicidality in patients with bipolar disorder. Psychiatry Res. 2020, 290, 113088. [CrossRef]

10. Kazhungil, F.; Mohandas, E. Management of obsessive-compulsive disorder comorbid with bipolar disorder. Indian J. Psychiatry 2016, 58, 259-269. [CrossRef]

11. Philip, J.; Janaki, R. Antidepressant-induced mania in obsessive compulsive disorder. Indian J. Psychiatry 2012, 54, 194-195. [CrossRef] [PubMed]

12. Raja, M.; Azzoni, A. Clinical management of obsessive-compulsive-bipolar comorbidity: A case series. Bipolar Disord. 2004, 6, 264-270. [CrossRef] [PubMed]

13. Maina, G.; Albert, U.; Rosso, G.; Bogetto, F. Olanzapine or lamotrigine addition to lithium in remitted bipolar disorder patients with anxiety disorder comorbidity: A randomized, single-blind, pilot study. J. Clin. Psychiatry 2008, 69, 609-616. [CrossRef] [PubMed]

14. Muneer, A. The treatment of adult bipolar disorder with aripiprazole: A systematic review. Cureus 2016, 8, e562. [CrossRef]

15. Yatham, L.N.; Kennedy, S.H.; Parikh, S.V.; Schaffer, A.; Bond, D.J.; Frey, B.N.; Sharma, V.; Goldstein, B.I.; Rej, S.; Beaulieu, S.; et al. Canadian Network for Mood and Anxiety Treatments (CANMAT) and International Society for Bipolar Disorders (ISBD) 2018 guidelines for the management of patients with bipolar disorder. Bipolar Disord. 2018, 20, 97-170. [CrossRef] [PubMed]

16. Malhi, G.S.; Bassett, D.; Boyce, P.; Bryant, R.; Fitzgerald, P.B.; Fritz, K.; Hopwood, M.; Lyndon, B.; Mulder, R.; Murray, G.; et al. Royal Australian and New Zealand College of Psychiatrists clinical practice guidelines for mood disorders. Aust. N. Z. J. Psychiatry 2015, 49, 1087-1206. [CrossRef]

17. Albert, U.; Marazziti, D.; Di Salvo, G.; Solia, F.; Rosso, G.; Maina, G. A systematic review of evidence-based treatment strategies for obsessive- compulsive disorder resistant to first-line pharmacotherapy. Curr. Med. Chem. 2018, 25, 5647-5661. [CrossRef]

18. Muscatello, M.R.; Bruno, A.; Pandolfo, G.; Micò, U.; Scimeca, G.; Romeo, V.M.; Santoro, V.; Settineri, S.; Spina, E.; Zoccali, R.A. Effect of aripiprazole augmentation of serotonin reuptake inhibitors or clomipramine in treatment-resistant obsessive-compulsive disorder: A double-blind, placebo-controlled study. J. Clin. Psychopharmacol. 2011, 31, 174-179. [CrossRef]

19. Pessina, E.; Albert, U.; Bogetto, F.; Maina, G. Aripiprazole augmentation of serotonin reuptake inhibitors in treatment-resistant obsessive-compulsive disorder: A 12-week open-label preliminary study. Int. Clin. Psychopharmacol. 2009, 24, 265-269. [CrossRef]

20. Sayyah, M.; Sayyah, M.; Boostani, H.; Ghaffari, S.M.; Hoseini, A. Effects of aripiprazole augmentation in treatment-resistant obsessive-compulsive disorder (a double blind clinical trial). Depress. Anxiety 2012, 29, 850-854. [CrossRef]

21. Sahraian, A.; Ehsaei, Z.; Mowla, A. Aripiprazole as an adjuvant treatment for obsessive and compulsive symptoms in manic phase of bipolar disorder: A randomized, double-blind, placebo-controlled clinical trial. Prog. Neuropsychopharmacol. Biol. Psychiatry 2018, 84, 267-271. [CrossRef] [PubMed]

22. Mataix-Cols, D.; Fernández de la Cruz, L.; Nordsletten, A.E.; Lenhard, F.; Isomura, K.; Simpson, H.B. Towards an international expert consensus for defining treatment response, remission, recovery and relapse in obsessive-compulsive disorder. World Psychiatry 2016, 15, 80-81. [CrossRef] [PubMed]

23. Fountoulakis, K.N.; Vieta, E. Efficacy and safety of aripiprazole in the treatment of bipolar disorder: A systematic review. Ann. Gen. Psychiatry 2009, 27, 8-16. [CrossRef] [PubMed]

24. Marcus, R.; Khan, A.; Rollin, L.; Morris, B.; Timko, K.; Carson, W.; Sanchez, R. Efficacy of aripiprazole adjunctive to lithium or valproate in the long-term treatment of patients with bipolar I disorder with an inadequate response to lithium or valproate monotherapy: A multicenter, double-blind, randomized study. Bipolar Disord. 2011, 13, 133-144. [CrossRef] [PubMed]

25. Blonde, L.; Khunti, K.; Harris, S.B.; Meizinger, C.; Skolnik, N.S. Interpretation and impact of real-world clinical data for the practicing clinician. Adv. Ther. 2018, 35, 1763-1774. [CrossRef] 